einer grösseren Vase mit eigenartiger Verzierung (Penis?), auf der vier aramäische(?) Zeichen stehen.

Zu dem Mabler'schen Artikel in Nr. I Sp. $3 \mathrm{ff}$. schreibt Herr Prof. Dr. W. Spiegelberg:

Der Text, welchen Herr Mahler zum Ausgangspunkt und zur Grundlage seiner chronologischen Berechnungen macht, liegt nicht nur in der Pyramide des Merenre (Z. $67 \mathrm{ff}$.) vor, sondern auch in den Pyramiden des Phiops I (Z. $97 \mathrm{ff}$.) und des Phiops II (Z. $47 \mathrm{ff}$.). Also derselbe Text findet sich bei 3 verschiedenen Königen! Schon damit erledigen sich alle aus dem Text gefolgerten chronologischen Schlüsse. Dieser selbst ist nun ganz missverstanden worden. Herr Mahler hat die erste Uebersetzung von Brugsch wieder abgedruckt, die in der damaligen Zeit (vor mehr als 20 Jahren) begreiflich war. Denn Brugsch stand zum ersten Male einem kleinen Text aus jener alten religiösen Litteratur gegenüber, die uns heute in so reicher Fiulle vorliegt. Jeder Aegyptolog hätte dem Astronomen Herrn Mahler mitteilen können, dass die Uebersetzung von Brugsch heute keine Existenzberechtigung mehr hat, und auf Masperos in allem Wesentlichen richtige Uebersetzung im Recueil V S. 172 und XII S. 60 verwiesen. Unter Berücksichtigung der metrischen Anordnung des poetischen Textes lautet diese so:

1 Siehe er kommt als Orion

Siehe Osiris kommt als Orion, Herr des Weines am schönen W: g feste.

3 Es sprach seine Mutter: Mein Erbe

I Es sprach sein Vater: Empfangeu vom Himmel, geboren von $\left.\operatorname{der} \mathrm{Dw}_{3}-\mathrm{t}^{1}\right)$ (Unterwelt o. ä.)

5 , Merenre

Empfangen hat dich der Himmel mit dem Orion

7 Geboren hat dich die Dw:-t mit dem Orion

8 Es lebt, wer da lebt, (?) nach dem Gebot der Götter

1) Der "Morgenstern" heisst dw: niv (masc.!) während hier von dwo:-t (fem.!) die Rede ist. Das Wort bezeichnet spïter allgemein die Unterwelt. Es bleibt aber zu untersuchen, ob dwos-t nicht, wie Jacoby einmal vermutete, in der alteren Litteratur den Nachthimmel - daher das Sterndeterminatir - im Gegensatz zu $p-t$ "Taghimmel " bezeichnete.
9 Da wirst ${ }^{1}$ ) leben ${ }^{2}$ )

${ }_{1}^{10} \mathrm{Du}$ wirst 1 ) emporsteigen mit dem Orion an der Ostseite des Himmels

${ }_{1}^{11} \mathrm{Da}$ wirst 1) hinabsteigen mit dem Orion an der Westseite des Himmels

12 Euer dritter ist die Sothis mit reinen Sitzen.

${ }_{1}^{13}$ Sie ist es, welche euch geleitet $z u$ den schönen Pfaden, welche im Himmel sind, im Gefilde ' $r{ }^{\prime}$ ".

Der Sinn dieses Textes ist völlig klar. Es wird hier wie so oft in den Pyramidentexten und auch in der späteren religiösen Litteratur geschildert, wie der selige Tote als Stern am Himmel weiter lebt. Ich denke und hoffe, dass auch der Astronom Herr Mahler mit mir der Ansicht sein wird, dass dieser Text kein „eminentes Interesse für die berechnende Chronologie der Könige des alten Reiches besitzt".

\section{Zur Transskriptionsfrage.}

Von Georg Hüsing.

In der Aufstellung dreier verschiedener Umschriftarten seitens des Herrn Herausgebers (OLZ $1903 \mathrm{Sp} .361$ ) vermisse ich die Rücksichtnahme auf die nichtsemitischen Sprachen, die in der OLZ vorkommen, und damit die Anbahnung eines A usgleichs, wie er nicht nur für geschichtliche Darstellungen, sondern gar oft auch für die Forschung selbst so erwünscht wäre. Den ärgsten Stein des Anstosses bildet hier die Wiedergabe des $\check{s}$ durch $\boldsymbol{s}$ bei den Indianisten, die um so störender wirken muss, als dass $s$ der semitischen Sprachen seinen annäherndsten Vertreter im $c$ der Indianisten hat. Es verdient jedenfalls an diesem Orte betont zu werden, dass es heute eine Richtung, die das $\breve{s}$ in dem Sanskrit durchzuführen bestrebt ist, bereits gibt; es ist also jedenfalls ein berechtigter Wunsch, dass z. B. in der OLZ, die ja einseitig indianistischen Interessen nicht dienen will, das $s$ (für $s \grave{s}$ ) verschwinde. Es kann sich ja so wie so in dieser Zeitschrift im wesentlichen nur um Fälle handeln, die unter die dritte der oben erwähnten Arten der Umschrift fallen, d. h. um Kunstausdrücke und Eigennamen. (Artikel und Besprechungen anderer Art stünden wohl in der OLZ nicht am richtigen Platze). Die

1) Die emphatische Verbalform.

3) Dabei ist zu beachten, dass " $n$, "leben* auch das "Aufgehen" der Sterne bezeichnet. 
Rüoksicht auf die Lautgruppen der Sanskritgrammatiker fällt also hier weg.

Weiter soll auf die Gesamtfrage hier nicht eingegangen werden. Die Dreiteilung scheint mir im übrigen den praktischen $\mathrm{Be}$ dürfnissen angemessen.

Einige Anmerknngen fordert die Aufstellung der dritten Art heraus.

Unter diese würde ja die Wiedergabe der Namen für volkstümlichere Veröffentlichungen fallen, und für diese sind Punkte und Häkchen möglichst zu vermeiden, sonst werden sie gelegentlich einfach weggelassen und es entstehen ungeahnte Namen.

Für diese $Z$ wecke dürfte es sich aber auch empfehlen, doch wenigstens in zwei Punkten assyrische und babylonische Formen auseinander zu halten, nämlich das assyrische $\check{s}$ in Namen durch $s$, das babylonische $m$ (wenigstens inlautend) durch $v$ wiederzugeben, also Schavasch, aber Samas zu schreiben, wo Babylonien und Assyrien geschieden werden. Letztere Form dürfte sich aber ihrer Einfachheit wegen auch als allgemeine empfehlen. So erhielten wir Formen wie Samsi-Adad, Sulmanasar, Sarrukin, die in der Praxis jedenfalls viel für sich hätten. Wir brauchen derartige Formen, sonst wird die Kunde vom alten Oriente niemals in weitere Kreise dringen, man betrachte nur die verzweifelten Purzelbäume in unseren Schullehrbüchern.

Handelt es sich um Fälle der dritten Art, die Schreibung des Namens in den Texten schwankt aber in den Vokalen dergestalt wie in Kunulua neben Kinalia, dann müssen wir so wie so annehmen, dass die wiederzugebenden Vokale weder $u$ noch $i$, weder $u$ noch $a$ waren, aber je zwischen beiden lagen. Richtiger als eine der beiden assyrischen Schreibungen wäre eine Umschrift Kïnolïa also gewiss, trifft sogar vielleicht die Sache, denn Liburna und Lubarna würden gleichermassen ein Lüborna spiegeln, jener Gau von Tabal aber Purütäs umschrieben werden können. (Es liegt wohl nahe, dabei an die Puru-hum-zi Teglatpilesars I za denken; sollte "hum" etwa einen Lautwert tim haben, so dass die Schreibung ungefähr ein "Purütenci“ meinte?) Derartige Namenformen gewährten also nicht nur Einfachheit und Bequemlichkeit, sie könnten auch zur Identifizierung und sprachlichen Erklärung ${ }^{1)}$ - es handelt sich um Hetiterland - führen helfen.

1) "Dam"-Damusa könnte als "Domü̈sa“ dem späteren Tomisa entsprechen, auch denke man an A-sir neben $A$-šsur in den ${ }_{n}$ kappadokischen" Urkunden.
Aehnlich liegt es z. B. mit dem Namen des bekannten Fürsten von Tabal, der A mbari-di, Am-ba-ri-is und Am-ri-is geschrieben wird. Wie lautet nun die praktische Form nach der dritten Art? Offenbar liegen zwei "Kasusformen" vor, eine auf $s$ und eine auf di. Die dritte Schreibung zeigt, dass das $a$ hinter dem $b$ nicht in den Namen gehört; man konnte "Amris" zu hören glauben, da ja zwischen $m$ und $r$ ein $b$ leicht erklingt, wo man sich gar keine Mühe gibt es zu bilden. Der Name heisst also Ambris, was der Assyrer nicht schreiben konnte, und wenn die verschiedene Schreibung uns heute eine Hülfe abgibt zur richtigen Lesung, für die Assyrer galt das gleiche, und so mag vielleicht die verschiedene Schreibung schon im Sinne des Schreibers den gleichen $Z$ weck verfolgt haben.

Wie in Am-ri-is das b unausgedrückt blieb, weil es sich von selbst einstellt, so in An-ša-an das $t$ zwischen $n$ und $s$. Die elamischen Texte schreiben den Namen mit dem gleichen Zeichen, das iranisches $\check{c}$ und $j$ ausdrückt, also tš und $d s ̌$ umschrieben werden könnte. Gemeint ist der scharfe Laut, wie aus der Möglichkeit hervorgeht, nach dem $n$ dafür ein $s$ einzusetzen. Volkstïmlich zu schreiben wäre also Antschan, und in der babylonischen Schreibung, die das gleiche Zeichen verwendet wie die elamische, ist $\mathbf{3}$ gemeint, nicht i. Zu umschreiben wäre also: nach 1. Art: An-sa-an, nach 2. am besten Ančan (da es kein babyl. Name ist), nach der 3. aber Antschan.

Mit diesen Beispielen, die leicht zu vermehren sind, will ich auf das eine hinaus: Während sich die beiden ersten Arten der Umschrift nach der Schrift richten müssen und dürfen, muss sich die dritte nach der Aussprache richten, so viel sie kann. Denn sie fasst naturgemäss die Schreibungen verschiedener Völker zusammen. "Xerxes" und "Ahasverus" sind gewissermassen Laut fïr Laut der gleiche Name. Hier kann man sich damit zu belfen suchen, dass man sich auf die grössere Verbreitung der griechischen Form beruft, um diese zu bevorzugen. Indessen ist auch das nur eine Modesache, die von selbst verschwinden würde, wenn wir aus der Zeit von 500-300 vor $\mathrm{Chr}$. reichliche einheimische Texte finden könnten, und zwar politisch-historischen Inhalts. Wären diese aber babylonisch, dann würde man weder die babylonische, noch die hebräische, noch die griechische Namenformen anwenden wollen, sondern sich nach einer Mittelform umsehen, und die "mittelste" 
wäre dann die einheimisch-iranische, d. h. diejenige, die der wirklichen Aussprache am nächsten steht. Von der Sprachform gehen ja doch die fremden Schreibungen aus, und nur die Sprachform bildet das natürliche Bindeglied.

Der Sprachform muss also die Umschrift der dritten Art zustreben. Seit Hugo Winckler

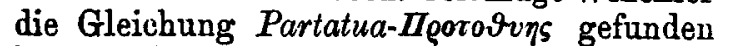
hat, ist der Ausdruck "Skythen " zweiwertig geworden, denn er bedeutet sowohl "Saken " wie das einzelne Volk. Die verschiedenen Namenformen des letzteren-in den Keilschrifttexten sind untereinander wie mit der griechischen nur zu vereinigen, wenn man sich fragt, welche ursprüngliche, eigentliche Form sie ausdrücken sollen. Richtig ist keine von allen. Sie ergeben aber ein "Skutscha" als Namen des Volkes ${ }^{1}$ ), und es dürfte kein Grund vorliegen, diesen in Umschrift der dritten Art zu vermeiden.

Auch dieser Fall soll nur wieder ein Beispiel seiner Gattung sein.

Es dürfte aber gerade jetzt, wo die Kunde vom alten Oriente beginnt, aus dem engsten Kreise durchzusickern, an der Zeit sein, gerade der dritten Art der Umschrift eine besondere Sorgfalt zuzuwenden. Das Hin- und Herschwanken erzeugt stets ein gewisses Misstrauen und reiohliche Missverständnisse. Bleibt aber die Entwickelung dem Zufalle überlassen, dann wird heute gebaut, was in zum Teil absehbarer 7eit mit Mühe wird wieder abgebrochen werden müssen. Wer sich je bemüht hat, ferner Stehende, an deren Gewinnung gelegen sein muss, zur Kenntnisnahme des bisher Erreichten zu bewegen, der wird auch die Erfahrung gemacht haben, dass die Namenformen durchaus keine gleichgültige Frage, vielmehr oft ein unüberwindliches Hindernis bilden. Mit einem Namen, den sie nicht drucken kann, weiss die Druckerei sich keinen Rat und ebenso wenig der Lehrer mit einem Namen, den er nicht auszusprechen weiss. Ich kenne Fälle, in denen sich die Auswahl des Stoffes lediglich darnach richtete, ob der Name "plausibel" war oder nicht.

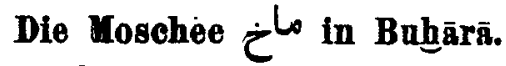 \\ Von Arthur Christensen.}

In der Buhārā-Chronik, welche mit dem Namen des Naršähi bezeichnet wird, findet

1) Auch hier meint also die Keilschrift ein s; nicht ;. sich (ed. Schefer p. 18-19) der folgende Passus :

Es hat in Buhārā eiuen Markt gegeben, den man $\left.b \bar{a} z \bar{a} r-i \quad M \bar{a} h-r \bar{u} z^{1}\right)$ genaunt. Zweimal des Jahres wurde hier ein eintägiger Markt gehalten, und an jedem Markttage wurden Götzenbilder verkauft zu einem Betrage von mehr als 50,000 Direm. Muhammad b. Gá far (d. h. Naršāhñ) hat in seinem Buche folgendes berichtet: „Dieser Markt hat bis zu unserer Zeit existiert, und als ich mich sehr wunderte, woher dieser Gebrauch stammte, frug ich die Alten und Greise in Buhärā, was der Anlass dazu wäre. Sie sagten: „Die Bewohner von Buhārā waren in alter Zeit Götzenanbeter, und ditser Handel [mit Götzenbildern] war bei ihuen eine feststehende Gewohnheit. Aus dieser Zeit entstammt der Götzenbilderverkauf, und der Markt hat sich bis jetzt erhalten." Abū-l-Hasan Nišàūirī berichtet in seinem Buche $\dot{H} a z a \bar{a} i n-e l-u \bar{l} \bar{u}$ : "In früheren Tagen war in Buhärā ein König namens Māh̆. Err befahl diesen Markt zu errichten, und er befahl Holzschneidern und Malern, jedes Jahr Götzenbilder zu varfertigen und sie an einem festgestellten Tage (also an den zwei jährlichen Māh-Tagen) herbeizubringen, um sie zu verkaufen. Und die Leute kauften sie, und jedesmal, wenn sie (d. h. die Götzenbilder) weggeworfen oder zerschlagen oder abgenutzt waren, kauften die Leute am Tage des Marktes ein neues und warfen das alte fort. Und da, wo heute die Moschee Māh ist, war eine Ebene am Rande des Flusses und viele Bäume, in deren Schatten der Markt gehalten wurde; und jener König kam und setzte sich auf seinen Thron an der Stelle, wo jetzt die Moschee Māh ist, damit die Leute mehr Lust bekamen Götzenbilder zu kaufen. Und ein jeder kaufte ein Bild für sich und nabm es mit nach Hause. Später wurde die Stelle in einen Feuertempel verwandelt, und am Tage des Marktes, wenn die Leute sich versammelten, gingen alle in diesem Feuertempel und beteten das Feuer an. Dieser Feuertempel stand bis zur Zeit des Islams. Als aber die Muslims die Oberhand gewannen, bauten sie an dieser Stelle jene Moschee, welche heute eine der vornehmsten Moscheen Buhārās ist."

Es handelt sich also hier um einen alten Kult, welcher später - vielleicht nur teil-

1) N. Lykoschin gibt in seiner russischen Uebersetzung des Narłähr (Istorija Buchary, Taschkent 1897) nur Bazar Moh. Es ist anch hier dio einzige Stelle in dem Schefer'schen Text, wo die vollere Form $b \bar{a} z \bar{a} r-i$ Măhr-rīz angeführt ist. 